CrossMark <click for updates

Cite this: Soft Matter, 2016, 12,228

Received 22nd July 2015, Accepted 8th October 2015 DOI: $10.1039 / \mathrm{c} 5 \mathrm{sm} 01814 \mathrm{e}$ www.rsc.org/softmatter

\title{
Buckling of paramagnetic chains in soft gels $\dagger$
}

\author{
Shilin Huang, ${ }^{a}$ Giorgio Pessot, ${ }^{b}$ Peet Cremer, ${ }^{b}$ Rudolf Weeber, ${ }^{c}$ Christian Holm, ${ }^{c}$ \\ Johannes Nowak, ${ }^{d}$ Stefan Odenbach, ${ }^{d}$ Andreas M. Menzel ${ }^{b}$ and \\ Günter K. Auernhammer*a
}

\begin{abstract}
We study the magneto-elastic coupling behavior of paramagnetic chains in soft polymer gels exposed to external magnetic fields. To this end, a laser scanning confocal microscope is used to observe the morphology of the paramagnetic chains together with the deformation field of the surrounding gel network. The paramagnetic chains in soft polymer gels show rich morphological shape changes under oblique magnetic fields, in particular a pronounced buckling deformation. The details of the resulting morphological shapes depend on the length of the chain, the strength of the external magnetic field, and the modulus of the gel. Based on the observation that the magnetic chains are strongly coupled to the surrounding polymer network, a simplified model is developed to describe their buckling behavior. A coarse-grained molecular dynamics simulation model featuring an increased matrix stiffness on the surfaces of the particles leads to morphologies in agreement with the experimentally observed buckling effects.
\end{abstract}

\section{Introduction}

Magneto-responsive hybrid gels (MRGs) have been attracting great attention due to their tunable elasticity, swelling properties and shape that can be remotely controlled by a magnetic field. They have potential applications as soft actuators, artificial muscles, as well as sensors ${ }^{1-3}$ and can serve as model systems to study the heat transfer in hyperthermal cancer treatment. ${ }^{4}$ Compared to other stimuli-responsive gels, MRGs have the advantage of fast response, controlled mechanical properties and reversible deformabilities. ${ }^{5-7}$ A typical MRG consists of a chemically cross-linked polymer network, swollen in a good solvent, and embedded magnetic particles. $^{5,8}$ The size of the magnetic particles can range from $\sim 10 \mathrm{~nm}$ to several $\mu \mathrm{m}^{7}$

The origin of the magneto-responsive behavior of MRGs is the magnetic interaction between the magnetic filler particles as well as their interaction with external magnetic fields. ${ }^{9,10}$ In a uniform magnetic field, paramagnetic particles can be polarized

\footnotetext{
${ }^{a}$ Max Planck Institute for Polymer Research, Ackermannweg 10, 55128 Mainz, Germany.E-mail: auhammer@mpip-mainz.mpg.de; Fax: +49 6131379 100; Tel: +49 6131379113

${ }^{b}$ Institut für Theoretische Physik II: Weiche Materie, Heinrich-Heine-Universität Düsseldorf, 40225 Düsseldorf, Germany ${ }^{c}$ Institute for Computational Physics, Universität Stuttgart, 70569 Stuttgart, Germany

${ }^{d}$ Chair of Magnetofluiddynamics, Measuring and Automation Technology, Institute of Fluid Mechanics, Technische Universität Dresden, 01069 Dresden, Germany

$\dagger$ Electronic supplementary information (ESI) available. See DOI: 10.1039/ c5sm01814e
}

and act as approximate magnetic dipoles. Depending on their mutual azimuthal configuration, the dipolar interactions can be either attractive or repulsive. In a liquid carrier, the dipolar interaction drives the magnetic particles to form chains and columns $^{11-14}$ aligning in the direction of the magnetic field. However, in a polymer gel, the particles cannot change their position freely. Instead, relative displacements of the particles, induced e.g. by changes in the magnetic interactions, lead to opposing deformations of the polymer network. As a result, the magnetic interactions can induce changes in the modulus of the gel. ${ }^{7,15}$ This magneto-elastic effect is well known to be related to the spatial distribution of the magnetic particles. ${ }^{16-21}$ For example, the modulus of anisotropic materials that contain aligned chain-like aggregates of magnetic filler particles ${ }^{15,22-24}$ can be significantly enhanced when an external magnetic field is applied along the chain direction. ${ }^{7}$ The anisotropic arrangement of particles also dominates the anisotropic magnetostriction effects. ${ }^{25-27}$

Different theoretical routes have been pursued to investigate the magneto-elastic effects of MRGs: macroscopic continuum mechanics approaches, ${ }^{28,29}$ mesoscopic modeling, ${ }^{16-19}$ and more microscopic approaches ${ }^{30-32}$ that resolve individual polymer chains. Theoretical routes to connect and unify these different levels of description have recently been proposed. ${ }^{33-35}$ The authors of ref. 34 show how the interplay between the mesoscopic particle distribution and the macroscopic shape of the sample affects the magneto-elastic effect. In addition to these factors, recent experiments and computer simulations also point out that a direct coupling between the magnetic particles and attached polymer chains can play another important role. , $30,31,36-39^{-39}$ 
An experimental model system showing a well-defined particle distribution and a measurable magneto-elastic effect can help to understand the magneto-elastic behavior of MRGs at different length scales. Projected into a two-dimensional plane, the distribution of magnetic particles in thin diluted MRGs can be detected using optical microscopy or light scattering methods. ${ }^{15,40}$ By combining these techniques with magnetic or mechanical devices, it is possible to observe the particle rearrangement when the MRG sample is exposed to a magnetic field or mechanical stimuli. ${ }^{15,41}$ For threedimensional (3D) characterization, X-ray micro-tomography has been used. ${ }^{23}$ Here we introduce another 3D imaging technique laser scanning confocal microscopy (LSCM). Compared to normal optical microscopy, LSCM is able to observe 3D structures and it has a better resolution. ${ }^{42}$ Compared to X-ray micro-tomography, LSCM is faster in obtaining a 3D image and easier to combine with other techniques for real-time investigation. ${ }^{43,44}$

We use LSCM to study the magneto-elastic effects of paramagnetic chains in soft gels. As a result, we find that the paramagnetic chains in soft gels (elastic modulus $<2 \mathrm{~Pa}$ ) under an oblique magnetic field show rich morphologies. Depending on the length of the chain, modulus of the gel and strength of an external magnetic field, the chains can rotate, bend, and buckle. The deformation field in the polymer network around the deformed paramagnetic chains can also be tracked. The result confirms that the chains are strongly coupled to the polymer network. A simplified model is developed to understand the magnetically induced buckling behavior of the paramagnetic chains in soft gels. In addition to serving as a model experimental system for studying the magneto-elastic effect of MRGs, our approach may also provide a new microrheological technique to probe the mechanical property of a soft gel. ${ }^{45}$ Furthermore, our results may be interesting to biological scientists who study how magnetosome chains interact with the surrounding cytoskeletal network in magnetotactic bacteria. ${ }^{46}$

\section{Materials and methods}

The elastic network was obtained by hydrosilation of a difunctional vinyl-terminated polydimethylsiloxane (vinyl-terminated PDMS, DMS-V25, Gelest Inc.) prepolymer with a SiH-containing cross-linker (PDMS, HMS-151, Gelest Inc.). Platinum(0)-1,3divinyl-1,1,3,3-tetramethyldisiloxane complex (Alfa Aesar) was used as a catalyst. A low-molecular-weight trimethylsiloxyterminated PDMS (770 $\mathrm{g} \mathrm{mol}^{-1}$, Alfa Aesar GmbH \& Co. KG, in the following "PDMS 770") served as a solvent that carried the polymer network and the paramagnetic particles. The paramagnetic particles were purchased from microParticles $\mathrm{GmbH}$. They were labeled with fluorophores (visible in LSCM). The materials consist of porous polystyrene spheres. Within the pores, nanoparticulate iron oxide was distributed rendering the particles superparamagnetic. To prevent iron oxide leaching, the particles had a polymeric sealing that also held the fluorophores. The particles had a diameter of $1.48 \pm 0.13 \mu \mathrm{m}$ (ESI, $\dagger$ Fig. S1a). We measured the magnetization curve (ESI, $\uparrow$ Fig. S1b) of the paramagnetic particles by a vibrating sample magnetometer
(VSM, Lake Shore 7407). We found about $20 \%$ deviations in the magnetic properties of the magnetic particles (e.g., magnetic moment, see ESI, $\dagger$ Fig. S2). In order to observe the deformation field in the polymer network, we used fluorescently labeled silica particles as tracers. They had a diameter of $830 \pm 50 \mathrm{~nm}$ and the surface was modified with $N, N$-dimethyl- $N$-octadel-3amino-propyltrimethoxysilylchloride.

After drying under vacuum at room temperature overnight, the paramagnetic particles were dispersed into PDMS 770. In some samples, tracer particles ( $3 \mathrm{wt} \%$ ) were also dispersed into PDMS 770 in this step. The prepolymer mixture was prepared with 9.1 wt\% vinyl-terminated PDMS and 90.9 wt $\%$ SiH-containing cross-linker. The prepolymer mixture (2.86 wt $\%$ ) was dissolved in PDMS 770, which contained the paramagnetic particles. Finally, by adding PDMS 770, which carried the catalyst, the concentration of the prepolymer mixture in the sol solution was carefully adjusted in the range from $2.74 \mathrm{wt} \%$ to $2.78 \mathrm{wt} \%$. This concentration range guaranteed the formation of soft gels with an elastic modulus lower than $10 \mathrm{~Pa}$ (see ESI, $\dagger$ Fig. S3). In the sol solution, the catalyst concentration was $0.17 \mathrm{wt} \%$, and the concentration of magnetic particles was $0.09 \mathrm{wt} \%$. The sol solution was agitated at $2500 \mathrm{rpm}$ with a Reax Control (Heidolph, Schwabach, Germany) for 2 min for homogenization, followed by ultrasonication (2 min, Transsonic $460 / \mathrm{H}$, Elma) to disperse the magnetic particles. Then the sol solution was introduced into a thin sample cell $(\sim 160 \mu \mathrm{m}$ thick and $\sim 1 \mathrm{~cm}$ wide) by capillary forces. The sample cells consisted of two No. 1 standard coverslips, separated by $\sim 160 \mu \mathrm{m}$ spacers. After sealing with two-component glue, the cells that contained the sol were exposed to a $100.8 \pm 0.5 \mathrm{mT}$ magnetic field. The paramagnetic particles aligned into chains along the direction of the applied magnetic field while the prepolymer was crosslinking. A visible reaction of the prepolymer occurred within $10 \mathrm{~min}$, and the rheological measurements showed that it took about $40 \mathrm{~min}$ to form a gel. Due to the low concentration of magnetic particles, the magnetic chains in the gel were well separated $(>30 \mu \mathrm{m})$. The length of the chains varied from a single particle up to about 170 particles. We stored the samples at ambient temperature for at least two weeks before testing. The modulus of the gels in the sample cells was characterized using microrheological techniques (see ESI, $\dagger$ Fig. S4). ${ }^{47}$

A home-built LSCM setup was used to observe the chain structure in the gel. ${ }^{43,44}$ We were able to analyze a sample of thickness of about $150 \mu \mathrm{m}$. A homogeneous magnetic field was attained by building Halbach magnetic arrays near the sample stage of the LSCM. $^{48}$ A 32-magnet array (Fig. 1a) was used to change the field direction while keeping the field strength constant (216.4 $\pm 1.1 \mathrm{mT}$, see ESI, $\dagger$ Fig. S5). Another 4-magnet Halbach array (see ESI, $\dagger$ Fig. S6) was used to change the field strength (up to $100.8 \pm 0.5 \mathrm{mT}$ ). The magnetic field was measured by a Lake Shore Model 425 Gaussmeter with a transverse probe.

To study the deformation of the PDMS gel around the magnetic chain, the magnetic field strength was increased from $0 \mathrm{mT}$ to $60.2 \pm 0.3 \mathrm{mT}$ in 8 steps ( $\sim 30 \mathrm{~min}$ per step). 3D images of the sample with randomly distributed tracer particles were recorded in every step. One isolated magnetic chain was chosen for further analysis. Thus, from the 3D images we extracted a 
(a)

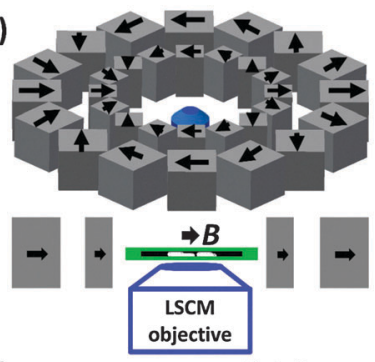

(b)

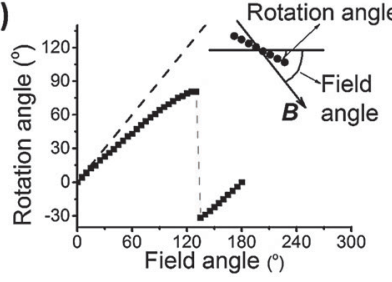

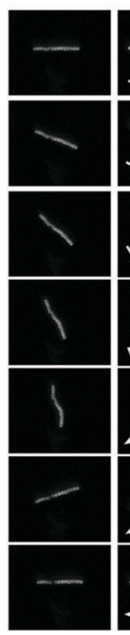

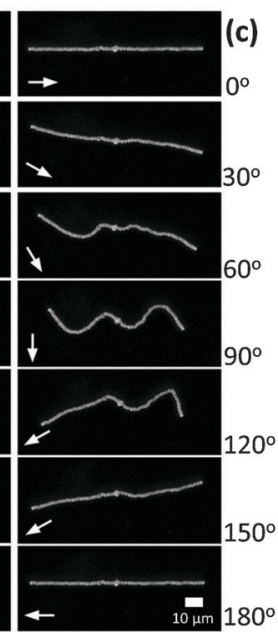

Fig. 1 (a) Laser scanning confocal microscopy (LSCM) was used to observe the morphologies of the paramagnetic chains in the soft gels. The Halbach magnetic array provided a homogeneous magnetic field (here $B=216.4 \pm 1.1 \mathrm{mT}$ ). This array could be rotated to change the orientation of the magnetic field. (b) The orientation of the magnetic field $\boldsymbol{B}$ was successively increased from $0^{\circ}$ to $180^{\circ}$ in 36 steps (square points). A magnetic chain of 15 particles rotated to follow the magnetic field, but the rotation angle was smaller than the orientation angle of $\boldsymbol{B}$ (dashed line). (c) Morphologies of magnetic chains in a soft gel changed when the orientation angle of $\boldsymbol{B}$ increased. The scale bar is $10 \mu \mathrm{m}$. The gel in (b) and (c) had an elastic modulus $\mathrm{G}^{\prime}$ of $0.25 \pm 0.06 \mathrm{~Pa}$

time series of $2 \mathrm{D}$ images that focused on this magnetic chain. From these 2D images, the trajectories of the embedded tracer particles were determined using the particle tracker plug-in developed on ImageJ software. ${ }^{49}$ The corresponding displacements of the tracers were deduced from the trajectories. Naturally, the tracer particles were stochastically distributed over the sample. We divided the images into a grid of small rectangles $\left(7.0 \times 5.5 \mu \mathrm{m}^{2}\right.$, containing, on average $\sim 3.5$ tracer particles) and determined the average displacement in each cell.

\section{Results}

In the absence of a magnetic field, the paramagnetic chains in a soft gel kept the aligned morphologies (ESI, $\dagger$ Section S1, Movie S1). When a magnetic field $(216.4 \pm 1.1 \mathrm{mT})$ was applied in the direction parallel to the chains (Fig. 1c, images for $0^{\circ}$ ), the paramagnetic chains still aligned with the original chain direction (horizontal). We changed the direction of the magnetic field step-by-step $\left(5^{\circ}\right.$ steps $)$ in the clockwise direction $(\sim 1 \mathrm{~min}$ between steps, quasi-static). We also define the orientation of the magnetic field $\boldsymbol{B}$ as the angle included between the magnetic field and the initial chain direction (see Fig. 1b). The left images of Fig. 1c show a short chain with 15 particles in a gel of elastic modulus $G^{\prime}$ of $0.25 \pm 0.06 \mathrm{~Pa}$. The chain rotated to follow the magnetic field. However, the rotation angle of the chain was smaller than the orientation angle of the magnetic field (Fig. 1b). This difference increased until the orientation of $\boldsymbol{B}$ reached $135^{\circ}$, where the chain flipped backward and had a negative angle. The chain again became parallel to the field when the orientation of

$\boldsymbol{B}$ increased to $180^{\circ}$. The morphology of the chain was the same at orientations of the magnetic field of $0^{\circ}$ and $180^{\circ}$ because of the superparamagnetic nature of the particles. Note that the chain was not straight at the intermediate angles (e.g., images for $60^{\circ}, 90^{\circ}$ and $120^{\circ}$ ). Instead it bended.

The images on the right-hand side of Fig. 1c show a longer chain with 59 particles in the same gel. When the orientation of $\boldsymbol{B}$ was $30^{\circ}$, the chain rotated and bended, with its two ends tending to point in the direction of the magnetic field. When the orientation of $\boldsymbol{B}$ was $60^{\circ}$, the chain wrinkled and started to buckle. A sinusoidal-shape buckling morphology was observed when the magnetic field was perpendicular to the original chain (orientation of the magnetic field of $90^{\circ}$, see ESI, $\dagger$ Section S1, Movie S2). When the orientation of $\boldsymbol{B}$ increased from $90^{\circ}$ to $120^{\circ}$, the left part of the chain flipped downward in order to follow the magnetic field. The right part flipped upward when the orientation of $\boldsymbol{B}$ increased from $120^{\circ}$ to $150^{\circ}$. Finally, when the field direction was again parallel to the original chain direction (orientation of the magnetic field of $180^{\circ}$ ), the chain became straight. The same rotation/buckling morphologies as in Fig. 1c could be observed when increasing the orientation of $\boldsymbol{B}$ from $180^{\circ}$ to $360^{\circ}$.

We also directly applied a perpendicular magnetic field to the paramagnetic chains in the soft gels. The paramagnetic chains showed different buckling morphologies (Fig. 2a) depending on the chain length. Fig. $2 \mathrm{~b}$ gives frequency counts of the different morphologies in the same sample $\left(G^{\prime}=0.25 \pm\right.$ $0.06 \mathrm{~Pa})$ under a magnetic field of $100.8 \pm 0.5 \mathrm{mT}$. In total 180 chains were observed. Longer chains tended to buckle with a higher number of half waves. Moreover, the distributions overlapped, implying that paramagnetic chains of identical length could have different morphologies under the perpendicular magnetic field.

These buckling morphologies are reminiscent of the buckling of paramagnetic chains in a liquid medium under a perpendicular magnetic field. ${ }^{50,51}$ The most stable morphology in the latter system
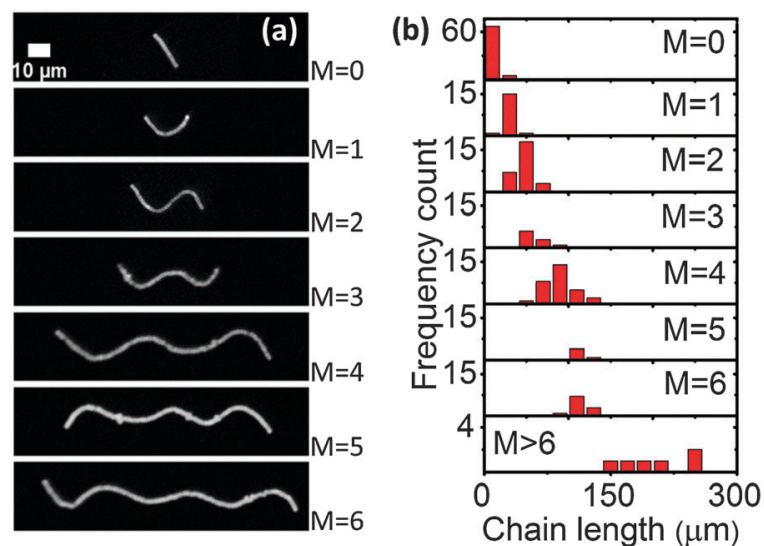

Fig. 2 (a) Different morphologies of paramagnetic chains in a soft gel $\left(G^{\prime}=0.25 \pm 0.06 \mathrm{~Pa}\right)$ under a perpendicular magnetic field $(100.8 \pm 0.5 \mathrm{mT})$. The original chain direction was horizontal, and the applied magnetic field was vertical. The scale bar is $10 \mu \mathrm{m}$. (b) Frequency count of different buckling morphologies in the same sample. $M$ is the number of half waves. 

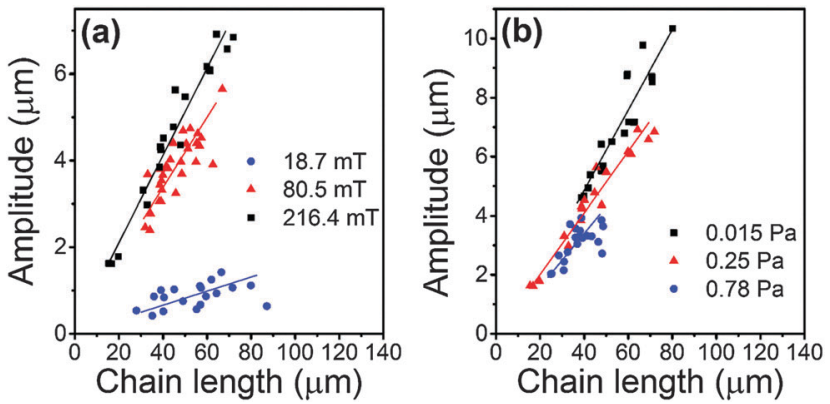

Fig. 3 Influence of chain length, strength of magnetic field and elastic modulus of the gel matrix on the amplitude of the S-shaped chains, observed when the magnetic field is applied perpendicularly to the initial chain orientation. (a) The elastic modulus of the gel was $0.25 \pm 0.06 \mathrm{~Pa}$, and the magnetic field strengths were $216.4 \pm 1.1 \mathrm{mT}$ (black squares), $80.5 \pm 0.4 \mathrm{mT}$ (red triangles), and $18.7 \pm 0.1 \mathrm{mT}$ (blue circles), respectively. (b) The magnetic field strength was $216.4 \pm 1.1 \mathrm{mT}$ and the elastic moduli of the gel were $0.015 \pm 0.005 \mathrm{~Pa}$ (black squares), $0.25 \pm 0.06 \mathrm{~Pa}$ (red triangles), and $0.78 \pm 0.22 \mathrm{~Pa}$ (blue circles), respectively. The solid lines are guides to the eye.

was a straight chain aligning along the magnetic field direction. However, in our system this morphology was not observed. Even the short chains showed a rotation angle smaller than the orientation of the magnetic field (e.g., Fig. 1b). The major difference between our experiments and ref. 50 and 51 was the nature of the surrounding medium. In our system, the polymer network around the paramagnetic chains impeded the rotation of the chains into the magnetic field direction (a more detailed discussion will be given below).

We used ImageJ software $\left(\mathrm{NIH}^{52}\right)$ to extract the skeletons of the chains that have 2 half waves (S-shaped). The amplitude of deflection or deformation of different chains was quantified by the square root of the mean square displacement, i.e. amplitude $=$ $\left(\left\langle y^{2}\right\rangle-\langle y\rangle^{2}\right)^{1 / 2}$, where $y$ measures the particle displacement along the field direction. The results are shown in Fig. 3. The amplitude increased with increasing chain length. At the same chain length, the amplitude tends to increase with increasing magnetic field strength (Fig. 3a; an example is also given in Fig. 4a) or with decreasing gel modulus (Fig. 3b).

The modulus dependence of the amplitude demonstrated that the polymer network around the paramagnetic chains impeded the chain deformations. Therefore, the deformation field within the polymer network plays an important role to understand the buckling of the chains. We thus added tracer particles into the gel matrix, and used their trajectories to record the deformation field around the paramagnetic chains. As shown in Fig. 4a, a linear paramagnetic chain buckled and formed an $\mathrm{S}$ shape in a perpendicular magnetic field. The amplitude increased with increasing field strength, while the contour length of the chain remained constant. The chain extension decreased along the original chain direction (horizontal direction) and increased along the perpendicular direction. Simultaneously, the polymer network around the chain followed the deformation (Fig. 4b) of the paramagnetic chain, both in the transverse and longitudinal directions. This confirmed that the paramagnetic chain is strongly coupled to the polymer network. Within our experimental

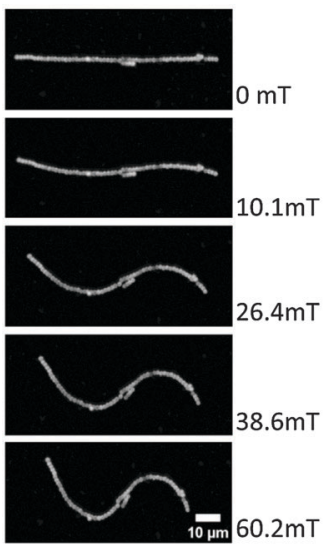

(a)

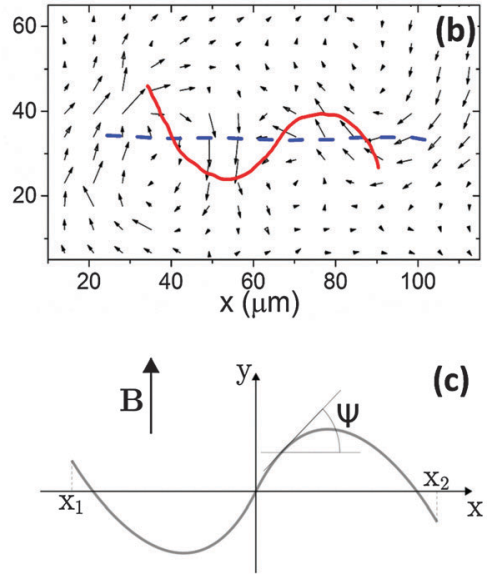

(c)

Fig. 4 (a) Influence of the magnetic field strength on a buckling chain. From top to bottom, the magnetic field strengths were $0 \mathrm{mT}, 10.1 \pm 0.1 \mathrm{mT}$, $26.4 \pm 0.1 \mathrm{mT}, 38.6 \pm 0.2 \mathrm{mT}$ and $60.2 \pm 0.3 \mathrm{mT}$, respectively. The modulus of this gel was about $0.01 \mathrm{~Pa}$. (b) Tracer particles were inserted into the gel matrix of the sample. Tracking these embedded tracer particles, the deformation field in the gel matrix was determined. The red solid line represents the skeleton of the magnetic chain shown in (a) for a field strength of $60.2 \pm 0.3 \mathrm{mT}$, and the dashed blue line indicates the original chain shape. (c) We modeled the paramagnetic chain in the elastic gel as a continuous object uniformly carrying dipolar magnetic moments. Without the magnetic field, the straight chain was oriented along the $x$-axis. Under a perpendicular magnetic field $\boldsymbol{B}$ (oriented along the $y$-axis), the magnetic chain deformed. The surrounding polymer network impeded the chain deformation.

resolution, the chain seemed to have a rigid non-slip contact to the surrounding network.

\section{Modeling}

We now turn to a qualitative description of the situation in the framework of a reduced minimal model. Theoretically capturing in its full breadth the problem of displacing rigid magnetic inclusions in an elastic matrix is a task of high complexity and enormous computational effort. ${ }^{53}$ We do not pursue this route in the following. Instead, we reduce our characterization to a phenomenological description in terms of the shape of the magnetic chain only. This is possible if the dominant modes of deformation of the surrounding matrix are reflected by the deformational modes of the magnetic chain.

Below, we assume that the chain is composed of identical spherical particles. In its undeformed state, the straight chain is located on the $x$-axis of our coordinate frame. The contour line of the deformed chain running through the particle centers is parameterized as $y(x)$, see Fig. $4 c$.

\subsection{Magnetic energy}

First, concerning the magnetic energy along the chain, we assume dipolar magnetic interactions between the particles. In the perpendicular geometry (Fig. 4c), the external magnetic field approximately aligns all dipoles along the $y$-axis. For simplicity, we only include nearest-neighbor magnetic interactions. In an 
infinite straight chain, this would result in an error given by a factor of $\zeta(3) \approx 1.2$, where $\zeta$ is the Riemann zeta function. ${ }^{33,54,55}$ Within our qualitative approach this represents a tolerable error. Replacing the magnetic interaction energy between the discrete magnetic particles by a continuous line integral and shifting the path of integration from the contour line of the chain to the $x$-axis, we obtain the magnetic interaction energy (see ESI, $\dagger$ Section S3.1)

$$
E_{\text {magn }}=W \int_{x_{1}}^{x_{2}} \frac{1}{\sqrt{1+y^{\prime}(x)^{2}}} \mathrm{~d} x,
$$

where $x_{1}$ and $x_{2}$ label the end points of the chain. The prefactor $W$ has the dimension of energy per unit length and is given by (see ESI, $\dagger$ Section S3.1)

$$
W \approx \frac{3 \mu_{0} m^{2}}{4 \pi d^{4}}
$$

where $\mu_{0}$ is the vacuum magnetic permeability, $m$ the magnetic moment of a single particle, and $d$ its diameter.

\subsection{Elastic bending energy}

Next, we need to include terms that provide a measure for the magnitude of the elastic deformation energy. To estimate the importance of different modes of the elastic matrix deformation, we analyze the experimentally determined displacement field around the distorted chain shown in Fig. 4b. For this purpose, we model the continuous matrix by a discretized spring network. ${ }^{19,56}$ Network nodes are set at the positions where the displacement field was tracked experimentally. The nodes are then connected by elastic springs. After that, we determine the normal modes of deformation of this network. ${ }^{56}$ Finally, we can decompose the experimentally observed deformation field in Fig. $4 \mathrm{~b}$ into these normal modes. Occupation numbers $\phi_{n}$ give the contribution of the $n$th mode to the overall deformation.

The four most occupied modes are shown in Fig. 5 . We find a major contribution of "oscillatory" modes, i.e. alternating up and down displacements along the central horizontal axis. Such oscillatory displacements of the matrix are connected to corresponding oscillatory displacements of the chain, see Fig. 4 b. A bending term of the form (see ESI, $\dagger$ Section S3.2)

$$
E_{\text {bend }}=C_{\mathrm{b}} \int_{x_{1}}^{x_{2}} \frac{\left[y^{\prime \prime}(x)\right]^{2}}{\left[1+y^{\prime}(x)^{2}\right]^{5 / 2}} \mathrm{~d} x
$$

becomes nonzero when such deformational modes occur and is therefore taken as a measure for their energetic contribution. In addition to that, we have experimental evidence that the chain itself shows a certain amount of bending rigidity (see ESI, $\dagger$ Fig. S7), possibly due to the adsorption of polymer chains on the surfaces of the magnetic particles. Similar indication follows from two-dimensional model simulations, see below.

\subsection{Elastic displacement energy}

The bending term does not energetically penalize rotations of a straight chain, see Fig. 2a for $M=0$. Yet, such rotations cost energy. Boundaries of the block of material are fixed, therefore any displacement of an inclusion induces a distortion of the
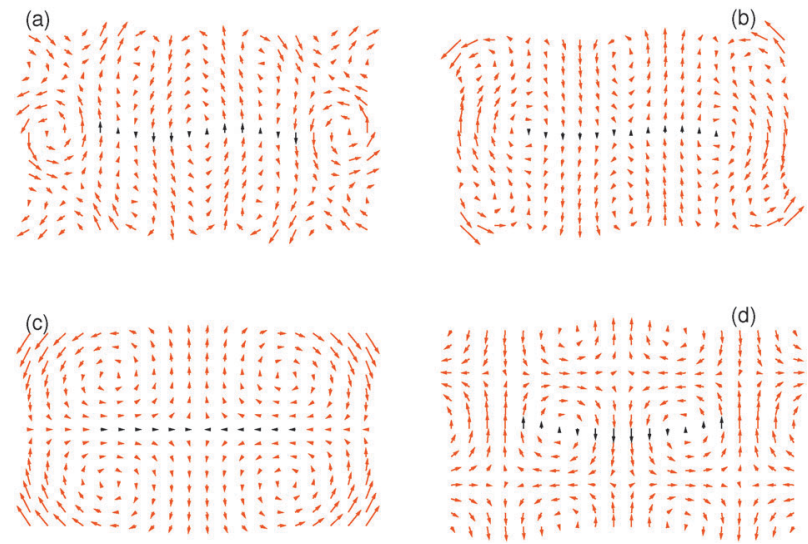

Fig. 5 The four most occupied normal modes of the deformation field in Fig. $4 \mathrm{~b}$ after projection to an elastic spring network, ordered by decreasing magnitude of contribution to the overall deformation. The normal modes (a), (b) and (d) are of an "oscillatory" type, whereas mode (c) represents a longitudinal contraction. Corresponding relative weights of the modes are $\phi_{(\mathrm{a})}{ }^{2}=0.095, \phi_{(\mathrm{b})}{ }^{2}=0.057, \phi_{(\mathrm{c})}{ }^{2}=0.055$, and $\phi_{(\mathrm{d})}{ }^{2}=0.051$, where we normalized the sum of the weights over all modes to unity. For better visualization, the overall amplitudes are rescaled as against the actual weights. The matrix region in close vicinity of the chain is indicated by black arrows.

surrounding gel matrix. We model this effect by a contribution (see ESI, † Section S3.3)

$$
E_{\mathrm{displ}}=C_{\mathrm{d}} \int_{x_{1}}^{x_{2}}[y(x)]^{2}\left[1+y^{\prime}(x)^{2}\right]^{3 / 2} \mathrm{~d} x .
$$

This term increasingly disfavors the rotations of longer straight chains, which reflects the experimental observations (see ESI, $\dagger$ Fig. S9).

Moreover, in Fig. $5 \mathrm{c}$ the third dominating mode of the matrix deformation corresponds to a contraction along the chain direction and an expansion perpendicular to it. We conjecture that this should be the dominating mode in the deformational far-field, yet this hypothesis needs further investigation. It is induced by chain deflections in $y$-direction, which imply a matrix contraction in $x$-direction (experimentally we observe that the chain length is conserved under deformations and that the individual magnetic particles remain in close contact). We simultaneously use $E_{\text {displ }}$ to represent the energetic contribution of this type of underlying matrix deformation.

\subsection{Energetic evaluation}

We now consider the resulting phenomenological model energy $E_{\mathrm{tot}}=E_{\mathrm{magn}}+E_{\mathrm{bend}}+E_{\mathrm{displ}}$. A standard procedure would consist of minimizing $E_{\text {tot }}$ with respect to the functional form of $y(x)$. Corresponding calculations and results are listed in the ESI, $\dagger$ Section S3.4. There are, however, severe limitations to this route in the present case. In contrast to several previous approaches in different contexts, ${ }^{57-61}$ our magnetic chains are of finite length and show significant displacements at their end points, see Fig. 2. Detailed knowledge of the boundary conditions of the deflection $y(x)$ and its derivatives at the end points of the chain would be necessary to determine the chain shape. 
Indeed the solutions become very sensitive to additional conditions (see ESI, $\dagger$ Section S3.4). In our case, the necessary additional boundary conditions depend on the interaction with the matrix. They are not accessible in the present reduced framework.

Therefore, we proceed in a different way. We use as an input for our calculations the experimental observations. The experimentally found chain shapes can to good approximation be represented by a polynomial form

$$
y(x)=S \prod_{m=0}^{M-1}(x-m b) \text { for } x_{1} \leq x \leq x_{2},
$$

where $M$ is again the number of half-waves, the prefactor $S$ sets the strength or amount of chain deformation and deflection, $b$ is the spacing between the nodes, and the interval $\left[x_{1}, x_{2}\right]$ follows from the experimental result of preserved chain length $L$,

$$
\int_{x_{1}}^{x_{2}} \sqrt{1+\left[y^{\prime}(x)\right]^{2}} \mathrm{~d} x=L .
$$

We prefer the polynomial form of eqn (5) to, for instance, a sinusoidal ansatz because it better reproduces the deformations of our finite-sized chain objects. In particular, the pronounced displacements of the chain ends, see e.g. Fig. 2a, are well captured by polynomial forms. Likewise, an experimentally observed tendency to smaller oscillation amplitudes inside longer chains is covered. Furthermore, rotations of short straight chains are readily included in this way.

Next, we insert eqn (5) into the above expressions for the energy and minimize with respect to $S, x_{1}$, and $x_{2}$ for a given $M$, with the constraint of constant length $L$, see eqn (6). The minimization was performed using Wolfram Mathematica minimization routines. ${ }^{62}$ Parameter values of the coefficients $C_{\mathrm{b}}$ and $C_{\mathrm{d}}$ are found by matching the resulting shapes to the corresponding experimental profiles (chain deformations for $G^{\prime}=0.25 \mathrm{~Pa}$ and magnetic field $B=100.8 \mathrm{mT}$ as in Fig. 2a, $M=2$, are used for this purpose). We obtain $C_{\mathrm{b}} \approx 0.01 \mathrm{~Wb}^{2}$ and $C_{\mathrm{d}} \approx 2 W / b^{2}$.

To illustrate how the energetic contributions vary under increasing preset deformation, we plot in Fig. 6 the energies for increasing $S$ for two fixed combinations of $M$ and $L$. The total energy $E_{\text {tot }}$ shows a global minimum in both panels, which we always observed for symmetric chain deformations. As expected, with increasing $S$ the magnetic energy decreases, whereas the deformation energies increase.

Next, we determine the minimal total energy as a function of chain length $L$ for different numbers of half-oscillations $M$, see Fig. 7. With increasing chain length $L$ the shapes that minimize the energy show an increasing number of half-waves $M$ in good agreement with the experimental data in Fig. 2 b.

Moreover, we quantify the amplitude of the chain deflection or deformation by

$$
\text { Amplitude }=\sqrt{\left\langle y^{2}\right\rangle-\langle y\rangle^{2}}, \quad\langle\cdot\rangle=\frac{\int_{x_{1}}^{x_{2}} \cdot \mathrm{d} x}{x_{2}-x_{1}} .
$$

Resulting values are plotted in Fig. 8. As mentioned above, we optimized the model parameters with respect to the experimental
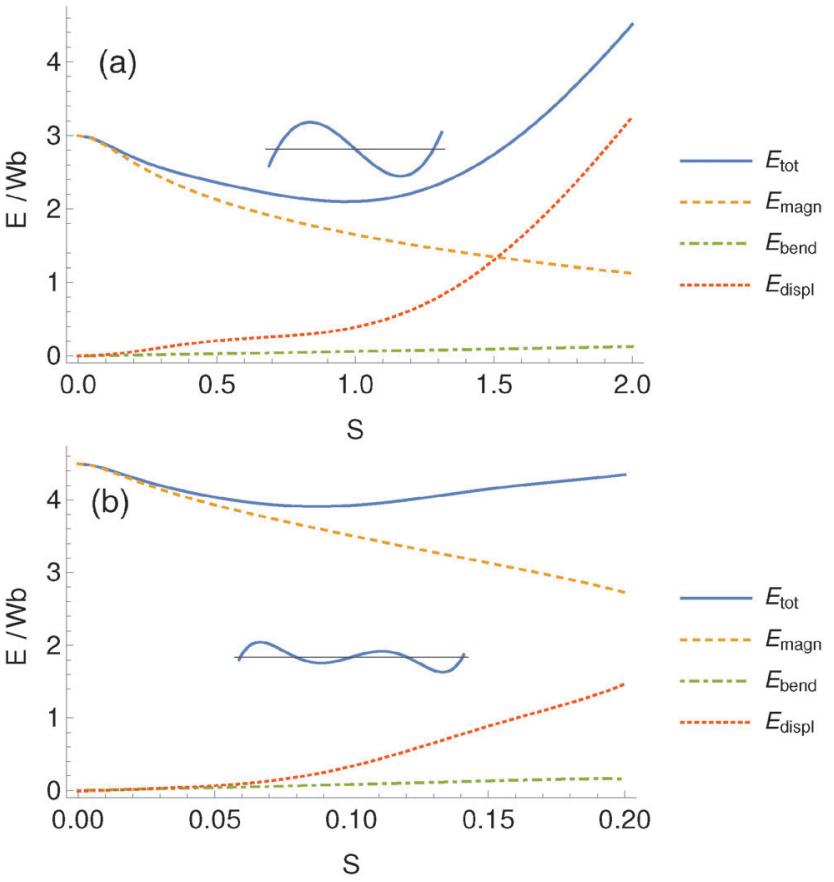

Fig. 6 Contributions to the total energy as a function of the amount $S$ of deformation and minimized with respect to $x_{1}$ and $x_{2}$ for a chain of the shape given by eqn (5). Here we show the cases (a) $M=2, L=3 b$ and (b) $M=4, L=4.5 b$. The total energy $E_{\text {tot }}$ has a global minimum as a function of $S$, which corresponds to the most stable chain shape. We always observed the global minimum for symmetric shapes.

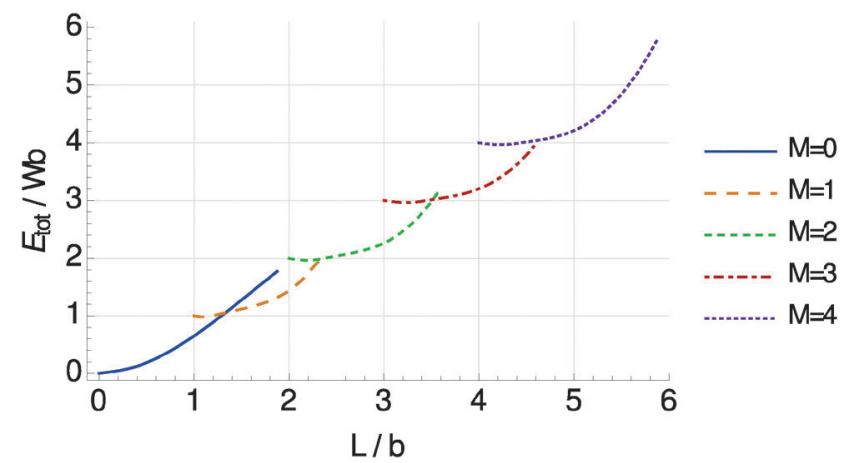

Fig. 7 Energies $E_{\text {tot }}$ of chain deformations of the shape given by eqn (5), minimized with respect to $S, x_{1}$, and $x_{2}$, as a function of chain length $L$ and number of half-oscillations $M$. Each curve describes a shape of $M$ half-waves with a minimum total length of $(M-1) b$. The resulting curves show crossing points from where the total energy for an increasing $L$ is lowered by bending one extra time (jumping to a higher $M$ ) rather than conserving the same shape.

data for a magnetic field intensity of $B=100.8 \mathrm{mT}$. We demonstrate in Fig. 8 that moderate variations of the magnetic field intensity only slightly affect our results: the brighter curves are obtained when multiplying the magnetic energy scale $W$ by a factor $\sim 1.42$, corresponding to an increased magnetic field intensity of approximately $B \sim 216 \mathrm{mT}$ (see ESI, $\uparrow$ Fig. S1b). This is in agreement with the experimental observations. We include in Fig. 8 the experimentally determined values for $B=80.5 \mathrm{mT}$ and $B=216.4 \mathrm{mT}$. Only a slight trend of increasing deflection amplitudes is found for this increase of magnetic field intensity. 


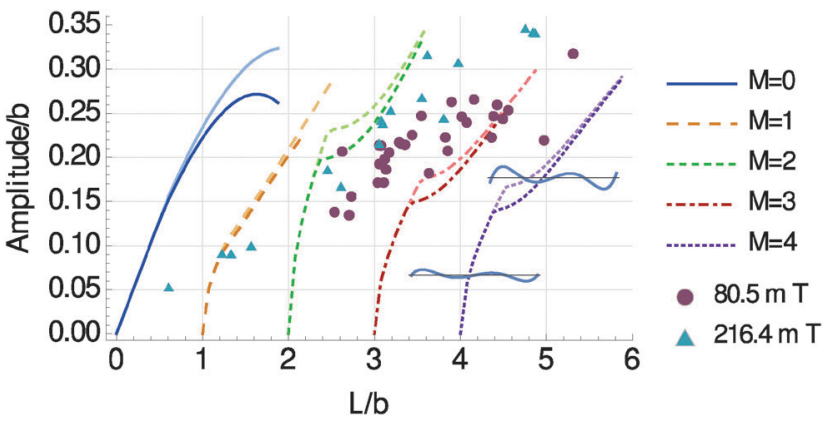

Fig. 8 Resulting deflection amplitudes of the chain deformation, calculated according to eqn (7). Darker curves represent the model parameters optimized with respect to the experimental shapes for a magnetic field intensity $B=100.8 \mathrm{mT}$. Brighter curves were obtained by increasing the magnetic energy scale $W$ by a factor $\sim 1.42$, which corresponds to an increased magnetic field intensity of $B \sim 216 \mathrm{mT}$ (see ESI, $\uparrow$ Fig. S1b), comparable with the triangular experimental data points. Both, model curves and included experimental data points, demonstrate that moderate variations of the magnetic field intensities only slightly affect the observed deflection and deformation amplitudes. The value of $b$ necessary to perform the analysis was determined from the $M=2$ experimental data as $b=12.6 \mu \mathrm{m}$. For $M \geq 2$ "kinks" appear in the curves, which arise from a change in the type of solution as illustrated by the insets: for each $M \geq 2$ curve, left of the kink the chain deformation shows nodes at the end points of the chain, i.e. $y\left(x_{1}\right) \simeq y\left(x_{2}\right) \simeq 0$ (lower left inset); right from the kink, these outer nodes are shifted to the inside of the chain (upper right inset). As seen from Fig. 7, the solutions left of the kinks are not energetically preferred.

Together, although the curves for $M=2$ in Fig. 8 slightly overshoot the data points, Fig. 7 and 8 are in good agreement with the experimental results. The amplitude of deflection and deformation is not observed to unboundedly increase with chain length $L$ in the experiments. Likewise, our model predicts that longer chains prefer to bend one extra time (switching to higher$M$ shape) rather than to show too large deflection amplitudes.

\section{Coarse-grained molecular dynamics simulation}

We also studied the buckling of the chain using two-dimensional coarse-grained molecular dynamics simulations by means of the ESPResSo software. ${ }^{63,64}$ A simple model was developed that allowed us to analyze the influence of particular interactions and material properties on the buckling effect. Here, we focus on the elasticity of the polymer matrix in the immediate vicinity of the magnetic particles.

By choosing the coarse-grained scale for our model, we ignore any chemical details but rather describe the system in terms of the magnetic particles as well as small pieces of polymer gel. As the buckling effect appears to be two-dimensional, and as the ground states for systems of dipolar particles have also been found to be two-dimensional, ${ }^{55}$ we use this dimensionality for our simulations. We study a chain of 100 magnetic particles with a significant amount of surrounding elastic matrix.

As in the analytical approach, the gel matrix is modeled by a network of springs. Here, however, we use a regular hexagonal mesh as a basis. To mimic the non-linear elastic behavior of polymers, we use a finitely extensible non-linear elastic spring potential (FENE-potential ${ }^{65}$ ) for the springs along the edges of the mesh. As a simple implementation of the finite compressibility, we introduce FENE-like angular potentials on the angles at the mesh points, with a divergence at $0^{\circ}$ and $180^{\circ}$ (see ESI, $\dagger$ Section S4.2). The magnetic particles are modeled as rigid spheres interacting by a truncated, purely repulsive Lennard-Jones potential, the so-called Weeks-Chandler-Andersen potential (see ESI, $\dagger$ Section S4.1). ${ }^{66}$ Their magnetic moment is assumed to be determined purely by the external magnetic field and to be constant throughout the simulation, i.e. we assume that the external field is significantly stronger than the field created by the particles. The magnetic moments are taken parallel to the external field and with a magnitude given by the experimentally observed magnetization curve. The coupling between the particles and the mesh is introduced in such a way, that under the volume occupied by a particle, the mesh does not deform, but rigidly follows the translational and rotational motion of the particle (see ESI, $\dagger$ Section S4.3). A local shear strain on the matrix can rotate a magnetic particle, but not its magnetic moment.

An important point is the elasticity of the polymer matrix in the immediate vicinity of the magnetic particles and, in particular, between two magnetic particles. We study two situations here, the first one including a stiffer region in the immediate vicinity of the particles, the second one without such a stiffer layer and directly jumping to the bulk elasticity. The stiffer layer, if imposed, is created using a spring constant larger by three orders of magnitude on those springs which originate from mesh sites within the particle volumes (see ESI, $\dagger$ Section S4.3). The angular potentials are unchanged.

A comparison between the cases with and without a stiffer layer of gel around the magnetic particles can be seen in Fig. 9. The images show a small part of the resulting configuration of magnetic particles and the surrounding mesh for a field applied perpendicular to the initial chain direction. Thus the magnetic moments of the particles are oriented perpendicular to the undistorted chain direction. This results in an energetically unfavorable parallel side-by-side configuration for the dipole moments. The energy can be reduced either by increasing the distance between the dipoles along the initial chain direction, or by moving dipoles perpendicularly to the initial chain direction so that they approach the energetically most favorable head-to-tail configuration. If the matrix is made stiffer immediately around the particles, and thus the contour length of the chain cannot change significantly, the re-positioning towards the head-to-tail configuration causes the buckling effect observed in the experiments (Fig. 9). When one assumes the matrix immediately around the magnetic particles to be as soft as in the bulk of the material, neighboring particles can move apart and the chain breaks up into individual particles or small columns perpendicular to the original chain direction. Additionally, a layer of increased stiffness also introduces a bending rigidity of the chain. In Fig. 10, the full chain and the surrounding matrix is shown for an external field of magnitude $216 \mathrm{mT}$, which from the experimental magnetization measurements corresponds to a magnetic moment of about $4.5 \times 10^{-14} \mathrm{~A} \mathrm{~m}^{2}$ (see ESI, $\dagger$ Fig. S1b). Due to the different 


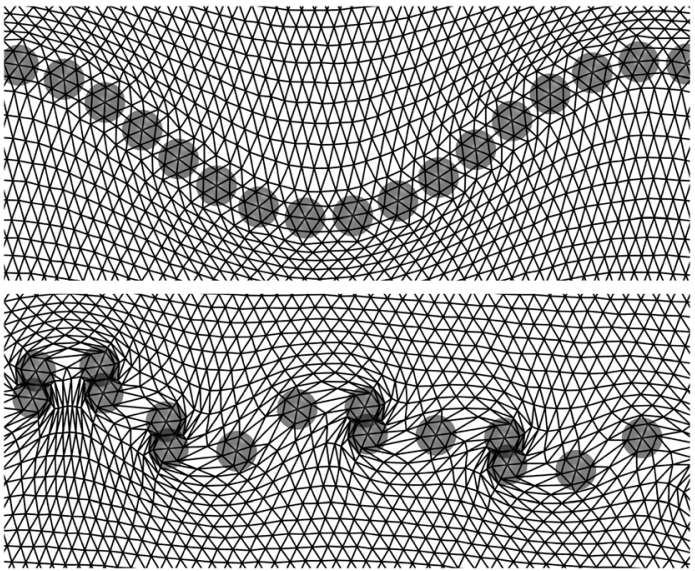

Fig. 9 Detailed view of the local deformations in the polymer mesh around the magnetic particles with a layer of increased stiffness (top) and without one (bottom) in the immediate vicinity of the particle surfaces. The external magnetic field of strength $216 \mathrm{mT}$ is applied in the vertical direction. When the boundary layer is assumed to be stiffer than the bulk (top), the buckling effect, as observed in the experiments, occurs. When the layer around the particles is soft (bottom), neighboring particles either form tight columns parallel to the field, or separate in the direction perpendicular to the field.

dimensionalities, the elastic modulus of the surrounding matrix could not be directly matched to the experimental system.

Actually, the amplitude of the chain oscillation increases when the external field is higher and induces larger dipole moments in the particles. This increases the tendency of the magnetic moments to approach the head-to-tail configuration, which in turn leads to a stronger deformation of the matrix. We note that the relative amplitude of the buckling along the chain is similar in the simulations (Fig. 9) and experiments (Fig. 2). The matrix surrounding the chain follows the chain oscillation with an amplitude that decreases over distance from the chain. Deviations may be expected from the deformational far-field in the experimental system due to the different dimensionalities of the systems.

In the $\mathrm{ESI}^{\dagger} \uparrow$ (Fig. S7) we show an experimental evidence for the existence of a stiff polymer layer around the magnetic particles. The sample was prepared at a concentration of prepolymer mixture well below the percolation threshold, i.e., some cross-linking of the polymer took place in the sample but no macroscopic gel was formed. When this cross-linking was done under an applied magnetic field, the particle chains stayed intact even after removal of the magnetic field (Fig. S7a, ESI $\dagger$ ). Hairpin or "S"-shape morphologies were observed when these chains were exposed to a magnetic field (Fig. S7b, ESI $\dagger$ ), indicating that they have a bending rigidity. ${ }^{50,51}$ Our interpretation of this behavior is that a stiff gel layer connects the particles and stabilizes the particle chains, even though no bulk gel is formed. As the magnetic particles have a good affinity for PDMS (e.g., the magnetic particles can be easily dispersed into PDMS), we conjecture that there is an adsorbed layer of polymer (i.e., PDMS prepolymer or cross-linker) on the surface of the magnetic particles. ${ }^{67}$ Therefore, the gel layer on the particles is denser and thus stiffer than in the bulk. Further study of the

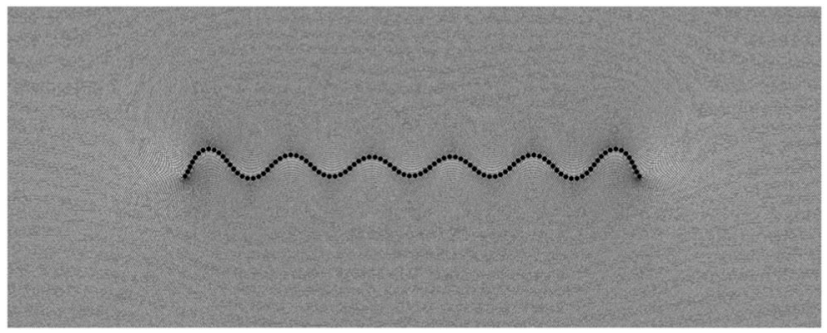

Fig. 10 Buckling chain of magnetic particles and the surrounding polymer mesh for an external field of magnitude $216 \mathrm{mT}$ pointing along the vertical direction. In this image, roughly one quarter of the full simulation area is shown. The surrounding matrix follows the chain oscillation with an amplitude that decreases over distance from the chain.

stiff polymer layer and its effect on the buckling behavior is under way.

Moreover, in Fig. S8 (ESI $\dagger$ ) we show that the buckling behavior of the magnetic chains can still be observed in the gel when we increase the elastic modulus to $170 \mathrm{~Pa}$ (for this purpose, carbonyl iron particles are used as magnetic filler particles). Our studies on that stiffer sample provide further evidence that the interaction between the magnetic particles and the polymer matrix in their close vicinity can play an important role in the magneto-elastic response of soft MRGs.

\section{Conclusions}

We have shown that paramagnetic chains in a soft polymer gel can buckle in a perpendicular magnetic field. The buckling morphology depends on the length of the chain, the strength of the magnetic field and the modulus of the gel. Longer chains form buckling structures with a higher number of half waves. Higher strengths of the magnetic field and a lower modulus of the gel matrix can lead to higher deformation amplitudes. The deformation field in the surrounding gel matrix confirms that the embedding polymer network is strongly coupled to the paramagnetic chain. A minimal magneto-elastic coupling model is developed to describe the morphological behavior of the paramagnetic chains in the soft gel under a perpendicular magnetic field. It shows that the chains deform in order to decrease the magnetic energy. This is hindered by the simultaneous deformation of the gel matrix, which costs elastic energy. Additionally, we have introduced a coarse-grained molecular dynamics simulation model, which covers both, the magnetic particles and the surrounding polymer mesh. In this model, the buckling of the chains can only be observed when the surface layer around the particles is assumed to be stiffer than the bulk of the gel. This prevents the chains from breaking up into columns oriented perpendicular to the initial chain direction or into isolated particles. These findings support the picture that the embedded magnetic chains themselves feature a certain bending rigidity, possibly due to the existence of a stiff polymer layer on the particle surfaces.

Since the magneto-elastic effect demonstrated and analyzed in this paper is pronounced, reversible, and controllable, it may 
be useful for designing micro-devices, e.g. micro-valves and pumps for microfluidic control. ${ }^{68}$ As the morphologies of the buckling paramagnetic chains are correlated with the modulus of the gel matrix, we may use them as mechanical probes for soft gels (similarly to active microrheology techniques). ${ }^{45}$ Moreover, our study may help to understand the physical interactions between the magnetic chains and the surrounding cytoskeleton network in magnetotactic bacteria. ${ }^{46}$ In our future study we will focus on how the interfacial coupling between the magnetic particles and the polymer network influences the local magnetoelastic coupling effect.

\section{Acknowledgements}

The authors acknowledge support by the Deutsche Forschungsgemeinschaft (DFG) through the SPP 1681 (SH and GKA acknowledge the project AU321/3-1; GP, PC and AMM acknowledge the project LO418/16-1; RW and $\mathrm{CH}$ acknowledge the project $\mathrm{HO}$ 1108/23-1; JN and SO acknowledge the project OD18/21). RW and $\mathrm{CH}$ acknowledge funding through the cluster of excellence EXC 310, SimTech at the University of Stuttgart. We thank Dr Peter Blümler for inspiring discussions on designing the Halbach magnetic arrays.

\section{References}

1 P. Ilg, Soft Matter, 2013, 9, 3465-3468.

2 R. L. Snyder, V. Q. Nguyen and R. V. Ramanujan, Smart Mater. Struct., 2010, 19, 055017.

3 K. Zimmermann, V. A. Naletova, I. Zeidis, V. Böhm and E. Kolev, J. Phys.: Condens. Matter, 2006, 18, S2973-S2983.

4 R. Hergt, S. Dutz, R. Müller and M. Zeisberger, J. Phys.: Condens. Matter, 2006, 18, S2919-S2934.

5 D. Szabó, G. Szeghy and M. Zrínyi, Macromolecules, 1998, 31, 6541-6548.

6 S. S. Abramchuk, D. A. Grishin, E. Y. Kramarenko, G. V. Stepanov and A. R. Khokhlov, Polym. Sci., Ser. A, 2006, 48, 138-145.

7 G. Filipcsei, I. Csetneki, A. Szilágyi and M. Zrínyi, Adv. Polym. Sci., 2007, 206, 137-189.

8 D. Collin, G. K. Auernhammer, O. Gavat, P. Martinoty and H. R. Brand, Macromol. Rapid Commun., 2003, 24, 737-741.

9 J. Faraudo, J. S. Andreu and J. Camacho, Soft Matter, 2013, 9, 6654-6664.

10 D. J. Griffiths, Introduction to Electrodynamics, Prentice-Hall, Upper Saddle River, NJ, 3rd edn, 1999, p. 282.

11 S. H. L. Klapp and M. Schoen, J. Chem. Phys., 2002, 117, 8050-8062.

12 S. H. L. Klapp, J. Phys.: Condens. Matter, 2005, 17, R525-R550.

13 G. P. Gajula, M. T. Neves-Petersen and S. B. Petersen, Appl. Phys. Lett., 2010, 97, 103103.

14 J. de Vicente, D. J. Klingenberg and R. Hidalgo-Alvarez, Soft Matter, 2011, 7, 3701-3710.

15 G. K. Auernhammer, D. Collin and P. Martinoty, J. Chem. Phys., 2006, 124, 204907.
16 D. S. Wood and P. J. Camp, Phys. Rev. E: Stat., Nonlinear, Soft Matter Phys., 2011, 83, 011402.

17 D. Ivaneyko, V. Toshchevikov, M. Saphiannikova and G. Heinrich, Condens. Matter Phys., 2012, 15, 33601.

18 Y. Han, W. Hong and L. E. Faidley, Int. J. Solids Struct., 2013, 50, 2281-2288.

19 G. Pessot, P. Cremer, D. Y. Borin, S. Odenbach, H. Löwen and A. M. Menzel, J. Chem. Phys., 2014, 141, 124904.

20 A. M. Menzel, Phys. Rep., 2015, 554, 1-46.

21 O. V. Stolbov, Y. L. Raikher and M. Balasoiu, Soft Matter, 2011, 7, 8484-8487.

22 G. Filipcsei, I. Csetneki, A. Szilágyi and M. Zrínyi, Magnetic Field-Responsive Smart Polymer Composites, Springer, Berlin, Heidelberg, 2007, vol. 206, pp. 137-189.

23 D. Günther, D. Y. Borin, S. Günther and S. Odenbach, Smart Mater. Struct., 2012, 21, 015005.

24 T. Borbáth, S. Günther, D. Y. Borin, T. Gundermann and S. Odenbach, Smart Mater. Struct., 2012, 21, 105018.

25 X. C. Guan, X. F. Dong and J. P. Ou, J. Magn. Magn. Mater., 2008, 320, 158-163.

26 K. Danas, S. V. Kankanala and N. Triantafyllidis, J. Mech. Phys. Solids, 2012, 60, 120-138.

27 A. Y. Zubarev, Soft Matter, 2013, 9, 4985-4992.

28 E. Jarkova, H. Pleiner, H. W. Müller and H. R. Brand, Phys. Rev. E: Stat., Nonlinear, Soft Matter Phys., 2003, 68, 041706.

29 S. Bohlius, H. R. Brand and H. Pleiner, Phys. Rev. E: Stat., Nonlinear, Soft Matter Phys., 2004, 70, 061411.

30 R. Weeber, S. Kantorovich and C. Holm, Soft Matter, 2012, 8, 9923-9932.

31 R. Weeber, S. Kantorovich and C. Holm, J. Magn. Magn. Mater., 2015, 383, 262.

32 A. V. Ryzhkov, P. V. Melenev, C. Holm and Y. L. Raikher, J. Magn. Magn. Mater., 2015, 383, 277.

33 A. M. Menzel, J. Chem. Phys., 2014, 141, 194907.

34 D. Ivaneyko, V. Toshchevikov, M. Saphiannikova and G. Heinrich, Soft Matter, 2014, 10, 2213-2225.

35 G. Pessot, R. Weeber, C. Holm, H. Löwen and A. M. Menzel, J. Phys.: Condens. Matter, 2015, 27, 325105.

36 L. Roeder, M. Reckenthäler, L. Belkoura, S. Roitsch, R. Strey and A. M. Schmidt, Macromolecules, 2014, 47, 7200-7207.

37 N. Frickel, R. Messing, T. Gelbrich and A. M. Schmidt, Langmuir, 2009, 26, 2839-2846.

38 N. Frickel, R. Messing and A. M. Schmidt, J. Mater. Chem., 2011, 21, 8466-8474.

39 R. Messing, N. Frickel, L. Belkoura, R. Strey, H. Rahn, S. Odenbach and A. M. Schmidt, Macromolecules, 2011, 44, 2990-2999.

40 I. Csetneki, G. Filipcsei and M. Zrínyi, Macromolecules, 2006, 39, 1939-1942.

41 H. N. An, J. Groenewold, S. J. Picken and E. Mendes, Soft Matter, 2014, 10, 997-1005.

42 M. Minsky, Scanning, 1988, 10, 128-138.

43 M. Roth, M. Franzmann, M. D’Acunzi, M. Kreiter and G. K. Auernhammer, 2011, arXiv preprint, arXiv:1106.3623.

44 M. Roth, C. Schilde, P. Lellig, A. Kwade and G. K. Auernhammer, Eur. Phys. J. E: Soft Matter Biol. Phys., 2012, 35, 124. 
45 C. Wilhelm, Phys. Rev. Lett., 2008, 101, 028101.

46 A. Körnig, J. Dong, M. Bennet, M. Widdrat, J. Andert, F. D. Müller, D. Schüler, S. Klumpp and D. Faivre, Nano Lett., 2014, 14, 4653-4659.

47 T. G. Mason, T. Gisler, K. Kroy, E. Frey and D. A. Weitz, J. Rheol., 2000, 44, 917-928.

48 H. Raich and P. Blümler, Concepts Magn. Reson., Part B, 2004, 23, 16-25.

49 I. F. Sbalzarini and P. Koumoutsakos, J. Struct. Biol., 2005, 151, 182-195.

50 C. Goubault, P. Jop, M. Fermigier, J. Baudry, E. Bertrand and J. Bibette, Phys. Rev. Lett., 2003, 91, 260802.

51 V. P. Shcherbakov and M. Winklhofer, Phys. Rev. E: Stat., Nonlinear, Soft Matter Phys., 2004, 70, 061803.

52 http://imagej.nih.gov/ij/.

53 C. Spieler, M. Kästner, J. Goldmann, J. Brummund and V. Ulbricht, Acta Mech., 2013, 224, 2453-2469.

54 M. A. Annunziata, A. M. Menzel and H. Löwen, J. Chem. Phys., 2013, 138, 204906.

55 T. Prokopieva, V. Danilov, S. Kantorovich and C. Holm, Phys. Rev. E: Stat., Nonlinear, Soft Matter Phys., 2009, 80, 031404.

56 M. Tarama, P. Cremer, D. Y. Borin, S. Odenbach, H. Löwen and A. M. Menzel, Phys. Rev. E: Stat., Nonlinear, Soft Matter Phys., 2014, 90, 042311.
57 I. Gelfand and S. Fomin, Calculus of variations, Prentice-Hall Inc., Englewood Cliffs, NJ, 1963.

58 G. W. Hunt, M. Wadee and N. Shiacolas, J. Appl. Mech., 1993, 60, 1033-1038.

59 B. Audoly, Phys. Rev. E: Stat., Nonlinear, Soft Matter Phys., 2011, 84, 011605.

60 H. Diamant and T. A. Witten, 2010, arXiv preprint arXiv: 1009.2487.

61 H. Diamant and T. A. Witten, Phys. Rev. Lett., 2011, 107, 164302.

62 Wolfram Research, Mathematica 10.0, 2014.

63 H. J. Limbach, A. Arnold, B. A. Mann and C. Holm, Comput. Phys. Commun., 2006, 174, 704-727.

64 A. Arnold, O. Lenz, S. Kesselheim, R. Weeber, F. Fahrenberger, D. Röhm, P. Košovan and C. Holm, Meshfree Methods for Partial Differential Equations VI, 2013, pp. 1-23.

65 H. R. Warner, Ind. Eng. Chem. Fundam., 1972, 11, 379-387. 66 J. D. Weeks, D. Chandler and H. C. Andersen, J. Chem. Phys., 1971, 54, 5237.

67 L. Cohen-Tannoudji, E. Bertrand, L. Bressy, C. Goubault, J. Baudry, J. Klein, J.-F. Joanny and J. Bibette, Phys. Rev. Lett., 2005, 94, 038301.

68 E. K. Fleischmann, H. L. Liang, N. Kapernaum, F. Giesselmann, J. Lagerwall and R. Zentel, Nat. Commun., 2012, 3, 1178. 